\title{
Fisioterapia en tiempos de COVID-19
}

\author{
Physiotherapy in times of COVID-19
}

La Fisioterapia/Terapia Física/Kinesiología es una profesión establecida en todo el mundo, que en tiempos de COVID-19 y cuarentena desempeña un rol importante en la atención del paciente desde las unidades criticas hasta su alta hospitalaria, e incluso con el uso de la tele-fisioterapia/tele-salud para las orientaciones de funcionabilidad en el hogar.

La formación del profesional en terapia física aborda el conocimiento del cuerpo y el movimiento del ser humano en su ciclo vital, en situación de salud y en condición de enfermedad; con base en los conocimientos científicos y tecnológicos de los diferentes sistemas que influyen en el movimiento corporal humano y sus alteraciones, alcanzando con ello las competencias que aseguren su óptima participación en lo personal, profesional y social; desempeñándose como un profesional en el ámbito de la salud pública y privada incorporado en el equipo de rehabilitación.

Profesionales que habilitan funcionabilidad a través de planes de intervención fisioterapéutico basado en un diagnóstico funcional y razonamiento clínico, permiten comprender y consolidar la toma de decisión e intervención en los procesos patológicos y manejo de la calidad de vida de los pacientes a través de procesos de recuperación y rehabilitación validos juiciosos y humanizados (1).

La Organización Panamericana de la Salud (OPS) ha lanzado un llamamiento a los donantes y socios y así conseguir el financiamiento para ampliar la capacidad de los países de las Américas para responder a COVID-19. La estrategia de respuesta descrita en este llamamiento tiene dos objetivos principales: frenar la transmisión del virus y mitigar el impacto en la salud del COVID-19 en la Región. Se necesitan US \$ 948 millones iniciales para apoyar los esfuerzos críticos de respuesta en los países que más necesitan ayuda hasta septiembre del año 2020. A medida que este brote evolucione, es probable que las necesidades aumenten y los requisitos financieros estimados se ajustarán en consecuencia (2).

Los fisioterapeutas que trabajan en centros de atención primaria de salud desempeñan un papel en el tratamiento de pacientes ingresados en el hospital con COVID-19 confirmado o sospechoso. La fisioterapia cardiorrespiratoria se centra en el tratamiento de afecciones respiratorias agudas y crónicas y tiene como objetivo mejorar la recuperación física después de una enfermedad aguda. La fisioterapia puede ser beneficiosa en el tratamiento respiratorio y la rehabilitación física de pacientes con COVID-19; su indicación se centra en los pacientes con secreciones abundantes de las vías respiratorias que no pueden eliminar de forma independiente, amerita una evaluación caso por caso para que las intervenciones se basen en los indicadores clínicos. A este grupo de pacientes hipersecretores o de tos ineficaz, también se incluyen los pacientes de alto riesgo y pacientes de comorbilidad existentes. La intervención en la UCI del fisioterapeuta es aplicar técnicas que permitan permeabilizar las vías respiratorias para pacientes ventilados y/o ubicar a los pacientes con insuficiencia respiratoria grave asociada con COVID-19, incluido el uso de la posición prona para optimizar la oxigenación (3).

Dado el tratamiento médico intensivo para algunos pacientes con COVID-19, incluida la ventilación pulmonar protectora prolongada, la sedación y el uso de agentes bloqueantes neuromusculares, los que ingresan en la UCI

Escuela de Tecnología Médica, Facultad de Medicina, Universidad Peruana Cayetano Heredia. Lima, Perú.

Jefe de Carrera, Profesor Auxiliar Departamento Académico de Tecnología Médica. 
pueden tener un alto riesgo de desarrollar debilidad adquirida en la UCI; esto puede empeorar su morbilidad y mortalidad. Por lo tanto, es esencial iniciar una rehabilitación temprana después de la fase aguda de la dificultad respiratoria para limitar la gravedad de la debilidad adquirida en la UCI y promover una recuperación funcional rápida. La fisioterapia tendrá un papel impprtante en proporcionar intervenciones de ejercicio, movilización y rehabilitación a los sobrevivientes de enfermedades críticas asociadas con COVID-19 con el fin de permitir un regreso funcional al hogar (3).

La Confederación mundial de Terapia Física (WCPT) ha emitido documentos relacionados al entorno hospitalario agudo para adultos y las recomendaciones para los fisioterapeutas en dos secciones: planificación y preparación de la fuerza laboral, incluida la evaluación para determinar las indicaciones para la fisioterapia; y la entrega de intervenciones de fisioterapia, incluidos los requisitos respiratorios y de movilización / rehabilitación, así como los requisitos de equipo de protección personal (EPP).

Se reconoce que las prácticas de Terapia Física/Fisioterapia/Kinesiología varían en todo el mundo, sin embargo, se sugiere usar estas recomendaciones, como alcance del ejercicio la práctica dentro del contexto local de cada país. Somos los profesionales incluidos en el proceso de recuperación estructurar guías de intervención basado en un contexto funcional y de inclusión a sus actividades laborales después de esta etapa.

Giuliana Condezo Casasola ${ }^{1, a}$

\section{Correspondencia:}

Giuliana Condezo Casasola

Correo electrónico: giuliana.condezo.c@upch.pe

\section{REFERENCIAS BIBLIOGRAFICAS}

1. Cristancho W. Fisioterapia en el Adulto Crítico con SDRA POR COVID-19. En: Manual Moderno Blog. Ciudad de Mexico: Manual Moderno; 24/04/2020. (citado el 25 de abril del 2020) Disponible en: https://www.manualmoderno.com/blog/post/ fisioterapia-en-el-adulto-critico-con-sdra-por-covid19fisioterapia-en-el-adulto-critico-con-sdra-po/?fbcli $\mathrm{d}=$ IwAR0GPTgLeNVMfO8SmIAvrylEIWqK4Xolx 9OkBy6bjuUEd2jrsW3H_p-X-KM
2. Pan American Health Organization. COVID-19 Situation in the Region of the Americas. Washington DC: Pan American Health Organization; 25/03/2020. (citado el 25 de abril del 2020) Disponible en: https://www.paho.org/en/topics/coronavirusinfections/coronavirus-disease-covid-19

3. Thomas P, Baldwin C, Bissett B, et al. Physiotherapy management for COVID-19 in the acute hospital setting: clinical practice recommendations. Journal of Physiotherapy. 2020. (Por publicarse.) DOI: 10.1016/j.jphys.2020.03.011 